\title{
Some special finite sums related to the three-term polynomial relations and their applications
}

\author{
Elif Cetin ${ }^{1 *}$, Yilmaz Simsek ${ }^{2}$ and Ismail Naci Cangul ${ }^{1}$
}

"Correspondence:

elifcetin@uludag.edu.tr

'Department of Mathematics,

Uludag University, Gorukle, Bursa

16059, Turkey

Full list of author information is

available at the end of the article

\section{空 Springer}

\begin{abstract}
We define some finite sums which are associated with the Dedekind type sums and Hardy-Berndt type sums. The aim of this paper is to prove a reciprocity law for one of these sums. Therefore, we define a new function which is related to partial derivatives of the three-term polynomial relations. We give a partial differential equation (PDE) for this function. For some special values, this PDE reduces the three-term relations for Hardy-Berndt sums (cf. Apostol and Vu in Pac. J. Math. 98:17-23, 1982; Berndt and Dieter in J. Reine Angew. Math. 337:208-220, 1982; Simsek in Ukr. Math. J. 56(10): 1434-1440, 2004; Simsek in Turk. J. Math. 22:153-162, 1998; Simsek in Bull. Calcutta Math. Soc. 85:567-572, 1993; Pettet and Sitaramachandraro in J. Number Theory 25:328-339, 1989), to the generalized Carlitz polynomials, which are defined by Beck (Diophantine Analysis and Related Fields, pp. 11-18, 2006), to the Gauss law of quadratic reciprocity (cf. Beck in Diophantine Analysis and Related Fields, pp. 11-18, 2006; Berndt and Dieter in J. Reine Angew. Math. 337:208-220, 1982; Simsek in Turk. J. Math. 22:153-162, 1998), and also to the well-known identity on the greatest integer function which was proved by Berndt and Dieter (J. Reine Angew. Math. 337:208-220, 1982), p.212, Corollary 3.5. Finally, we prove the reciprocity law for an $n$-variable new sum which is related to the Dedekind type and Hardy-Berndt type sums. We also raise some open questions on the reciprocity laws of our new finite sums.
\end{abstract}

MSC: Primary 11F20; secondary 11C08

Keywords: Hardy-Berndt sums; Dedekind sums; three-term polynomial relations; greatest integer function; $Y(h, k)$ sums

\section{Introduction}

The Dedekind sums are very useful in analytic number theory, in combinatorial theory and also in other branches of mathematics. That is, these sums arise in many areas of mathematics and also mathematical physics. Recently, there are many papers on the Dedekind sums which are related to elliptic modular functions, geometry (lattice point enumeration in polytopes, topology (signature defects of manifolds), algorithmic complexity (pseudo random number generators), character theory, the family of zeta functions, the Bernoulli functions, and other special functions. In 1877, Dedekind gave, under the modular transformation, an elegant functional equation for the Dedekind eta function, which contains the Dedekind sums.

O2014 Cetin et al.; licensee Springer. This is an Open Access article distributed under the terms of the Creative Commons Attribution License (http://creativecommons.org/licenses/by/2.0), which permits unrestricted use, distribution, and reproduction in any medium, provided the original work is properly cited. 
On the other hand, Berndt [1], Goldberg [2] and also Simsek [3] gave, under the modular transformation, other elegant functional equations for the theta functions, which contain six different arithmetic sums (Hardy-Berndt sums). These sums are also related to the Dedekind sums and other special functions which have been mentioned before. Motivated largely by a number of recent investigations of the Dedekind sums and the Hardy-Berndt sums, we introduce and investigate various properties of a certain new family of finite arithmetic sums. We are ready to summarize our results in detail as follows.

In this section, some elementary properties and definitions on the Dedekind sums, the Hardy-Berndt sums, and the Simsek sum are given. In Section 2, we define some new finite arithmetic sums which are associated with the Dedekind sums, the Hardy-Berndt sums, and Simsek's sum. We gave reciprocity laws for one of these sums. We also raise two open questions for the reciprocity laws. In the last section, we give a PDE for three-term polynomial relations. We give many applications for this PDE, which are related to the Dedekind-Rademacher sums, the Hardy-Berndt sums, and other finite arithmetic sums. Finally, by using this equation we give a proof of the reciprocity law of our new sums.

In the customary notation, we have

$$
((x))= \begin{cases}x-[x]-\frac{1}{2}, & \text { if } x \text { is not an integer } \\ 0, & \text { otherwise }\end{cases}
$$

where $[x]$ denotes the largest integer $\leq x(c f .[1-17]$, and the references cited in each of these earlier works). Let $n$ be a positive natural number and $\alpha$ be a real number, then

$$
(\alpha)_{n}= \begin{cases}\prod_{k=1}^{n}(\alpha+k-1), & \text { if } n \geq 1, \\ 1, & \text { if } n=0,\end{cases}
$$

and

$$
[\alpha]_{n}= \begin{cases}\prod_{k=1}^{n}([\alpha]+k-1), & \text { if } n \geq 1 \\ 1, & \text { if } n=0\end{cases}
$$

The Dedekind sum $s(h, k)$, arising in the theory of the Dedekind eta function, is defined by

$$
s(h, k)=\sum_{j=1}^{k-1}\left(\left(\frac{j}{k}\right)\right)\left(\left(\frac{h j}{k}\right)\right),
$$

where $h$ is an integer and $k$ is a positive integer ( $c f$. [1-17], and the references cited in each of these earlier works). The most important property of Dedekind sums is the following reciprocity theorem: If $h$ and $k$ are coprime positive integers, then

$$
s(h, k)+s(k, h)=-\frac{1}{4}+\frac{1}{12}\left(\frac{h}{k}+\frac{k}{h}+\frac{1}{h k}\right) .
$$

A proof of (1) was given by Apostol [4] and the references cited in each of these earlier works. 
If $h$ and $k$ are integers with $k>0$, the Hardy-Berndt sums are defined by

$$
\begin{aligned}
& S(h, k)=\sum_{j=1}^{k-1}(-1)^{j+1+\left[\frac{j h}{k}\right]} \\
& s_{1}(h, k)=\sum_{j=1}^{k-1}(-1)^{\left[\frac{j h}{k}\right]}\left(\left(\frac{j}{k}\right)\right), \\
& s_{2}(h, k)=\sum_{j=1}^{k-1}(-1)^{j}\left(\left(\frac{j}{k}\right)\right)\left(\left(\frac{j h}{k}\right)\right), \\
& s_{3}(h, k)=\sum_{j=1}^{k-1}(-1)^{j}\left(\left(\frac{j h}{k}\right)\right), \\
& s_{4}(h, k)=\sum_{j=1}^{k-1}(-1)^{\left[\frac{j h}{k}\right]}, \\
& s_{5}(h, k)=\sum_{j=1}^{k-1}(-1)^{j+\left[\frac{h j}{k}\right]}\left(\left(\frac{j}{k}\right)\right) .
\end{aligned}
$$

For $s_{5}(h, k)$, the equality below also holds true:

$$
s_{5}(h, k)=\frac{1}{k} \sum_{j=1}^{k-1} j(-1)^{j+\left[\frac{h j}{k}\right]}
$$

when $h$ and $k$ are odd [8]. Besides, the following equations will be very useful for the remaining sections [11]:

$$
\begin{aligned}
& \sum_{j=1}^{b-1}(-1)^{j+\left[\frac{c j}{b}\right]}\left(\frac{j}{b}\right)=s_{5}(c, b)-\frac{1}{2} S(c, b), \\
& \sum_{j=1}^{c-1}(-1)^{j+\left[\frac{b j}{c}\right]}\left(\frac{j}{c}\right)=s_{5}(b, c)-\frac{1}{2} S(b, c) .
\end{aligned}
$$

The reciprocity law for the $s_{5}(h, k)$ is given by the following theorem.

Theorem 1 Let $h$ and $k$ be coprime positive integers. If $h$ and $k$ are odd, then

$$
s_{5}(h, k)+s_{5}(k, h)=\frac{1}{2}-\frac{1}{2 h k}
$$

(cf. $[1,2,5,8,10,17]$ and the references cited in each of these earlier works). In the following theorem, Sitaramachandraro [17] showed that the Hardy-Berndt sum $s_{5}(h, k)$ can be expressed explicitly in terms of Dedekind sums.

Theorem 2 Let $h$ and $k$ be coprime positive integers. If $h+k$ is even, then

$$
s_{5}(h, k)=-10 s(h, k)+4 s(2 h, k)+4 s(h, 2 k),
$$


and if $h+k$ is odd, then

$$
s_{5}(h, k)=0 .
$$

The next theorem will be useful for the further sections.

Theorem 3 If both $h$ and $k$ are odd and $(h, k)=1$, then

$$
S(h, k)=S(k, h)=0 .
$$

A proof of this theorem was given by Apostol in [5]. In [16], Simsek defined a new sum related to the sums $s_{5}(h, k)$ as follows:

Let $h$ and $k$ be integers with $(h, k)=1$

$$
Y(h, k)=4 k \sum_{j=1}^{k-1}(-1)^{j+\left[\frac{h j}{k}\right]}\left(\left(\frac{j}{k}\right)\right) .
$$

The reciprocity law for the Simsek sum $Y(h, k)$ is given by $(c f$. [16, p.5, Theorem 4])

$$
h Y(h, k)+k Y(k, h)=2 h k-2 .
$$

\subsection{Three-term polynomial relations for the Hardy sums}

Here, two and three-term polynomial relations, which were studied thoroughly in [11] and [14], are recalled. In $[6,7,11]$, and [14] some new theorems on three-term relations for the Hardy sums were found by applying derivative operator to the three-term polynomial relation. Throughout this section, we assume that $a, b$, and $c$ are pairwise coprime positive integers and $a^{\prime}, b^{\prime}$, and $c^{\prime}$ satisfy

$$
a a^{\prime} \equiv 1 \quad(\bmod b), \quad b b^{\prime} \equiv 1 \quad(\bmod c), \quad \text { and } \quad c c^{\prime} \equiv 1 \quad(\bmod a) .
$$

The following corollary was given by Pettet and Sitaramachandrarao [11].

Corollary 1 (Three and two-term polynomial relations) If $a, b$, and c are pairwise coprime positive integers, then

$$
\begin{aligned}
& (u-1) \sum_{x=1}^{a-1} u^{x-1} v^{\left[\frac{b x}{a}\right]} w^{\left[\frac{c x}{a}\right]}+(v-1) \sum_{y=1}^{b-1} v^{y-1} w^{\left[\frac{c y}{b}\right]} u^{\left[\frac{a y}{b}\right]}+(w-1) \sum_{z=1}^{c-1} w^{z-1} u^{\left[\frac{a z}{c}\right]} v^{\left[\frac{b z}{c}\right]} \\
& \quad=u^{a-1} v^{b-1} w^{c-1}-1 \\
& (u-1) \sum_{x=1}^{a-1} u^{x-1} v^{\left[\frac{b x}{a}\right]}+(v-1) \sum_{y=1}^{b-1} v^{y-1} u^{\left[\frac{a y}{b}\right]}=u^{a-1} v^{b-1}-1 .
\end{aligned}
$$


The identity (9) is originally due to Berndt and Dieter [7]. The next corollary, which is equivalent to (9), was first established by Carlitz [9].

Corollary 2 [9] If $a$ and $b$ are coprime positive integers, then

$$
(u-1) \sum_{x=1}^{b-1} u^{b-x-1} v^{\left[\frac{a x}{b}\right]}-(v-1) \sum_{y=1}^{a-1} v^{a-y-1} u^{\left[\frac{b y}{a}\right]}=u^{b-1}-v^{a-1} .
$$

We need the following relations, which were proved by Pettet and Sitaramachandrarao [11]:

$$
\begin{aligned}
& s_{1}\left(c a^{\prime}, b\right)=\sum_{y=1}^{b-1}(-1)^{y+\left[\frac{c y}{b}\right]}\left(\left(\frac{a y}{b}\right)\right), \\
& s_{4}\left(b c^{\prime}, a\right)=\sum_{x=1}^{a-1}(-1)^{\left[\frac{b x}{a}\right]+\left[\frac{c x}{a}\right]} \\
& s_{3}\left(a b^{\prime}, c\right)=\sum_{z=1}^{c-1}(-1)^{z+\left[\frac{b z}{c}\right]}\left(\left(\frac{a z}{c}\right)\right), \\
& s_{2}\left(c a^{\prime}, b\right)=\sum_{y=1}^{b-1}(-1)^{y}\left(\left(\frac{c y}{b}\right)\right)\left(\left(\frac{a y}{b}\right)\right), \\
& s_{1}\left(b c^{\prime}, a\right)=\sum_{x=1}^{a-1}(-1)^{\left[\frac{b x}{a}\right]}\left(\left(\frac{c x}{a}\right)\right), \\
& s_{3}\left(a b^{\prime}, c\right)=\sum_{z=1}^{c-1}(-1)^{\left[\frac{b z}{c}\right]}\left(\left(\frac{a z}{c}\right)\right),
\end{aligned}
$$

and also

$$
s_{5}\left(c b^{\prime}, a\right)=\sum_{x=1}^{a-1}(-1)^{x+\left[\frac{c x}{a}\right]}\left(\left(\frac{b x}{a}\right)\right) .
$$

\section{New sums involving the functions $[x]$ and $((x))$}

In this section, we define some new finite sums which are related to not only the functions $[x]$ and $((x))$, but also the Dedekind sums, the Hardy-Berndt sums, the Simsek sum $Y(h, k)$, and the other finite sums. We also investigate the reciprocity laws of these sums. We also ask two open questions for these reciprocity laws.

Definition 1 Let $a_{1}, a_{2}, \ldots, a_{n}$ be pairwise coprime positive integers. We define the following sums $Y_{n-1}\left(a_{1}, \ldots, a_{n-1} ; a_{n}\right), B_{n-1}\left(a_{1}, \ldots, a_{n-1} ; a_{n}\right)$ and $C\left(a_{1}, a_{2}, \ldots, a_{n-1} ; a_{n} ; k\right)$, respectively:

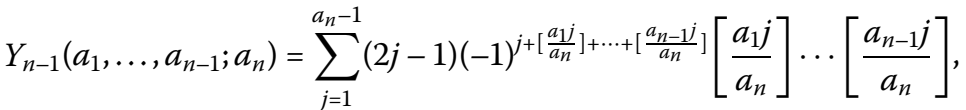

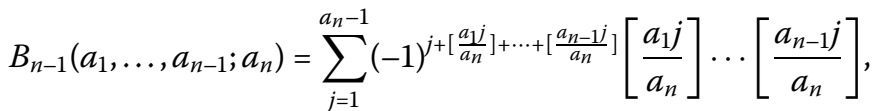




$$
C\left(a_{1}, a_{2}, \ldots, a_{n-1} ; a_{n} ; k\right)=\sum_{j=1}^{a_{n}-1} j^{k}(-1)^{j+\left[\frac{a_{1} j}{a_{n}}\right]+\cdots+\left[\frac{a_{n-1} j}{a_{n}}\right]},
$$

where $n \geq 2$ is a positive integer.

Substituting $n=2$ and $k=2$ into (17), we have

$$
\begin{aligned}
& Y_{1}\left(a_{1} ; a_{2}\right)=\sum_{j=1}^{a_{2}-1}(2 j-1)(-1)^{j+\left[\frac{a_{1} j}{a_{2}}\right]}\left[\frac{a_{1} j}{a_{2}}\right], \\
& B_{1}\left(a_{1} ; a_{2}\right)=\sum_{j=1}^{a_{2}-1}(-1)^{j+\left[\frac{a_{1} j}{a_{2}}\right]}\left[\frac{a_{1} j}{a_{2}}\right],
\end{aligned}
$$

and

$$
C\left(a_{1} ; a_{2} ; 2\right)=\sum_{j=1}^{a_{2}-1} j^{2}(-1)^{j+\left[\frac{a_{1} j}{a_{2}}\right]} .
$$

Combining the function $[x]=x-((x))-\frac{1}{2}$ with (18) and (19), we get

$$
\begin{aligned}
Y_{1}\left(a_{1}, a_{2}\right)= & -\left(a_{1}+a_{2}\right) s_{5}\left(a_{1}, a_{2}\right)+C_{1}\left(a_{1}, a_{2}\right)-2 C_{2}\left(a_{1}, a_{2}\right) \\
& +\frac{2 a_{1}}{a_{2}} C\left(a_{1} ; a_{2} ; 2\right)-\frac{1}{2} S\left(a_{1}, a_{2}\right)
\end{aligned}
$$

and

$$
B\left(a_{1}, a_{2}\right)=a_{1} s_{5}\left(a_{1}, a_{2}\right)-C_{1}\left(a_{1}, a_{2}\right)+\frac{1}{2} S\left(a_{1}, a_{2}\right),
$$

where

$$
C_{1}\left(a_{1}, a_{2}\right)=\sum_{j=1}^{a_{2}-1}(-1)^{j+\left[\frac{a_{1} j}{a_{2}}\right]}\left(\left(\frac{a_{1} j}{a_{2}}\right)\right)
$$

and

$$
C_{2}\left(a_{1}, a_{2}\right)=\sum_{j=1}^{a_{2}-1} j(-1)^{j+\left[\frac{a_{1} j}{a_{2}}\right]}\left(\left(\frac{a_{1} j}{a_{2}}\right)\right) .
$$

Thus, our new definitions are related to the Hardy-Berndt sums and also the Simsek sum $Y\left(a_{1}, a_{2}\right)$. The reciprocity laws for the special finite sums, that is, the Dedekind type sums, the Hardy-Berndt type sums, and the Simsek sum, are very important. Therefore, we are ready to give the reciprocity law of the sums $Y_{n-1}\left(a_{1}, a_{2}, \ldots, a_{n-1} ; a_{n}\right)$ by the following theorem.

Theorem 4 Let $n$ be a natural number with $n \geq 2$ and $a_{1}, a_{2}, \ldots, a_{n}$ be positive integers, relatively prime in pairs. Then we have 


$$
\begin{aligned}
& Y_{n-1}\left(a_{2}, a_{3}, \ldots, a_{n} ; a_{1}\right)+Y_{n-1}\left(a_{1}, a_{3}, \ldots, a_{n} ; a_{2}\right)+\cdots+Y_{n-1}\left(a_{1}, a_{2}, \ldots, a_{n-1} ; a_{n}\right) \\
& \quad=\prod_{m=1}^{n}(-1)^{a_{m}-1}\left(a_{m}-1\right) .
\end{aligned}
$$

Let $a_{1}, a_{2}, \ldots, a_{n}$ be positive integers, relatively prime in pairs. Then we define the following sums:

$$
Y S_{k, n}\left(a_{1}, \ldots, a_{n-1} ; a_{n}\right)=\sum_{j=1}^{a_{n}-1} \frac{j^{k}}{a_{n}^{k}}\left(\left(\frac{a_{1} j}{a_{n}}\right)\right)\left(\left(\frac{a_{2} j}{a_{n}}\right)\right) \cdots\left(\left(\frac{a_{n-1} j}{a_{n}}\right)\right) .
$$

Substituting $k=1$ and $n=2$ into (20), we arrive at the Dedekind sums

$$
Y S_{1,2}\left(a_{1} ; a_{2}\right)=s\left(a_{1} ; a_{2}\right)=\frac{1}{a_{2}} \sum_{j=1}^{a_{2}-1} j\left(\left(\frac{a_{1} j}{a_{2}}\right)\right),
$$

where $\left(a_{1} ; a_{2}\right)=1$.

Substituting $n=2$ into (20), we obtain

$$
Y S_{k, 2}\left(a_{1} ; a_{2}\right)=\sum_{j=1}^{a_{2}-1} \frac{j^{k}}{a_{2}^{k}}\left(\left(\frac{a_{1} j}{a_{2}}\right)\right)
$$

\section{Open questions}

(1) For $n \geq 2$, find the reciprocity laws of the sums $Y S_{k, n}\left(a_{1}, a_{2}, \ldots, a_{n-1} ; a_{n}\right)$ and $B_{n-1}\left(a_{2}, a_{3}, \ldots, a_{n} ; a_{1}\right)$. That is, find

$$
Y S_{k, n}\left(a_{2}, a_{3}, \ldots, a_{n} ; a_{1}\right)+Y S_{k, n}\left(a_{1}, a_{3}, \ldots, a_{n} ; a_{2}\right)+\cdots+Y S_{k, n}\left(a_{1}, a_{2}, \ldots, a_{n-1} ; a_{n}\right)
$$

and

$$
B_{n-1}\left(a_{2}, a_{3}, \ldots, a_{n} ; a_{1}\right)+B_{n-1}\left(a_{1}, a_{3}, \ldots, a_{n} ; a_{2}\right)+\cdots+B_{n-1}\left(a_{1}, \ldots, a_{n-1} ; a_{n}\right) .
$$

(2) For $k>1$, find the reciprocity law of $C\left(a_{1}, a_{2}, \ldots, a_{n-1} ; a_{n} ; k\right)$. That is, evaluate

$$
C\left(a_{2}, a_{3}, \ldots, a_{n} ; a_{1} ; k\right)+C\left(a_{1}, a_{3}, \ldots, a_{n} ; a_{2} ; k\right)+\cdots+C\left(a_{1}, \ldots, a_{n-1} ; a_{n} ; k\right) .
$$

\section{PDE for the Carlitz polynomials and their applications}

In this section, we study on the Carlitz polynomials and their properties $(c f .[6,7,9,11,14]$, and the references cited in each of these earlier works). We find a PDE for this polynomial. We give many applications for this PDE, which are related to the Dedekind-Rademacher sums, the Hardy-Berndt sums, and the other finite sums. In [6], Beck defined generalized the Carlitz polynomials as follows.

Definition 2 (The Carlitz polynomial) $c\left(u_{1}, u_{2}, \ldots, u_{n} ; a_{1}, a_{2}, \ldots, a_{n}\right)$, where $u_{1}, u_{2}, \ldots, u_{n}$ are indeterminants and $a_{1}, a_{2}, \ldots, a_{n}$ are positive integers, is defined as the polynomial




Theorem 5 (Berndt-Dieter) If $a_{1}, a_{2}, \ldots, a_{n}$ are pairwise relatively prime positive integers, then

$$
\begin{aligned}
\left(u_{1}-1\right) c\left(u_{1}, u_{2}, \ldots, u_{n} ; a_{1}, a_{2}, \ldots, a_{n}\right)+\left(u_{2}-1\right) c\left(u_{2}, u_{3}, \ldots, u_{n}, u_{1} ; a_{2}, a_{3}, \ldots, a_{n}, a_{1}\right) \\
\quad+\cdots+\left(u_{n}-1\right) c\left(u_{n}, u_{1}, \ldots, u_{n-1} ; a_{n}, a_{1}, \ldots, a_{n-1}\right) \\
\quad+u_{1}^{a_{1}-1} u_{2}^{a_{2}-1} \cdots u_{n}^{a_{n}-1}-1
\end{aligned}
$$

Proof of Theorem 5 was given by Beck in [6]. Now we will give a new definition:

Definition 3 Let $u, v, w$ be indeterminants. Let $a, b, c$ be positive integers, relatively prime in pairs, and let $k_{1}, k_{2}, k_{3}$ be positive natural numbers, which are the orders of the derivatives of $u, v, w$, respectively. Then the polynomial $\mathcal{F}\left(u, v, w ; a, b, c ; k_{1}, k_{2}, k_{3}\right)$ is defined by

$$
\begin{aligned}
\mathcal{F}\left(u, v, w ; a, b, c ; k_{1}, k_{2}, k_{3}\right)= & k_{1} \sum_{x=1}^{a-1}(x-1)_{k_{1}-1}\left[\frac{b x}{a}\right]_{k_{2}}\left[\frac{c x}{a}\right]_{k_{3}} u^{x} v^{\left[\frac{b x}{a}\right]} w^{\left[\frac{c x}{a}\right]} \\
& +(u-1) \sum_{x=1}^{a-1}(x-1)_{k_{1}}\left[\frac{b x}{a}\right]_{k_{2}}\left[\frac{c x}{a}\right]_{k_{3}} u^{x-1} v^{\left[\frac{b x}{a}\right]} w^{\left[\frac{c x}{a}\right]} \\
& +k_{2} \sum_{y=1}^{b-1}(y-1)_{k_{2}-1}\left[\frac{a y}{b}\right]_{k_{1}}\left[\frac{c y}{b}\right]_{k_{3}} v^{y} w^{\left[\frac{c y}{b}\right]} u^{\left[\frac{a y}{b}\right]} \\
& +(v-1) \sum_{y=1}^{b-1}(y-1)_{k_{2}}\left[\frac{a y}{b}\right]_{k_{1}}\left[\frac{c y}{b}\right]_{k_{3}} v^{y-1} w^{\left[\frac{c y}{b}\right]} u^{\left[\frac{a y}{b}\right]} \\
& +k_{3} \sum_{z=1}^{c-1}(z-1)_{k_{3}-1}\left[\frac{a z}{c}\right]_{k_{1}}\left[\frac{b z}{c}\right]_{k_{2}} w^{z} u^{\left[\frac{a z}{c}\right]} v^{\left[\frac{b z}{c}\right]} \\
& +(w-1) \sum_{z=1}^{c-1}(z-1)_{k_{3}}\left[\frac{a z}{c}\right]_{k_{1}}\left[\frac{b z}{c}\right]_{k_{2}} w^{z-1} u^{\left[\frac{a z}{c}\right]} v^{\left[\frac{b z}{c}\right]}
\end{aligned}
$$

where $k_{1}, k_{2}$, and $k_{3}$ are not zero simultaneously.

By using (8), we derive the following theorem, which is very important and valuable to obtain some new and old identities related to the function [·], the Dedekind sums, the Hardy-Berndt sums, and the Simsek sum $Y(h, k)$.

Theorem 6 The following identity holds true:

$$
\mathcal{F}\left(u, v, w ; a, b, c ; k_{1}, k_{2}, k_{3}\right)=(a-1)_{k_{1}}(b-1)_{k_{2}}(c-1)_{k_{3}} u^{a-1} v^{b-1} w^{c-1} \text {. }
$$

Proof By using (8), we have

$$
\begin{aligned}
P(u, v, w)= & (u-1) \sum_{x=1}^{a-1} u^{x-1} v^{\left[\frac{b x}{a}\right]} w^{\left[\frac{c x}{a}\right]}+(v-1) \sum_{y=1}^{b-1} v^{y-1} w^{\left[\frac{c y}{b}\right]} u^{\left[\frac{a y}{b}\right]} \\
& +(w-1) \sum_{z=1}^{c-1} w^{z-1} u^{\left[\frac{a z}{c}\right]} v^{\left[\frac{b z}{c}\right]}=u^{a-1} v^{b-1} w^{c-1}-1 .
\end{aligned}
$$


First we take the partial derivative of $P(u, v, w)$ with respect to $u$, then we have

$$
\begin{aligned}
\frac{\partial P(u, v, w)}{\partial u}= & \sum_{x=1}^{a-1} u^{x-1} v^{\left[\frac{b x}{a}\right]} w^{\left[\frac{c x}{a}\right]}+(u-1) \sum_{x=1}^{a-1}(x-1) u^{x-2} v^{\left[\frac{b x}{a}\right]} w^{\left[\frac{c x}{a}\right]} \\
& +(v-1) \sum_{y=1}^{b-1}\left[\frac{a y}{b}\right] v^{y-1} w^{\left[\frac{c y}{b}\right]} u^{\left[\frac{a y}{b}\right]-1}+(w-1) \sum_{z=1}^{c-1}\left[\frac{a z}{c}\right] w^{z-1} u^{\left[\frac{a z}{c}\right]-1} v^{\left[\frac{b z}{c}\right]} \\
= & (a-1) u^{a-2} v^{b-1} w^{c-1}
\end{aligned}
$$

Now, we take the partial derivative of $\frac{\partial P(u, v, w)}{\partial u}$ with respect to $u$, and we get

$$
\begin{aligned}
\frac{\partial^{2} P(u, v, w)}{\partial u^{2}}= & 2 \sum_{x=1}^{a-1}(x-1) u^{x-2} v^{\left[\frac{b x}{a}\right]} w^{\left[\frac{c x}{a}\right]} \\
& +(u-1) \sum_{x=1}^{a-1}(x-1)(x-2) u^{x-3} v^{\left[\frac{b x}{a}\right]} w^{\left[\frac{c x}{a}\right]} \\
& +(v-1) \sum_{y=1}^{b-1}\left[\frac{a y}{b}\right]\left(\left[\frac{a y}{b}\right]-1\right) v^{y-1} w^{\left[\frac{c y}{b}\right]} u^{\left[\frac{a y}{b}\right]-2} \\
& +(w-1) \sum_{z=1}^{c-1}\left[\frac{a z}{c}\right]\left(\left[\frac{a z}{c}\right]-1\right) w^{z-1} u^{\left[\frac{a z}{c}\right]-2} v^{\left[\frac{b z}{c}\right]} \\
= & (a-1)(a-2) u^{a-3} v^{b-1} w^{c-1} .
\end{aligned}
$$

If we continue this process with the mathematical induction method, taking partial derivative of $\frac{\partial^{k_{1}-1} P(u, v, w)}{\partial u^{k_{1}-1}}$ with respect to $u$, then we get

$$
E(u, v, w)=\frac{\partial^{k_{1}} P(u, v, w)}{\partial u^{k_{1}}}=(a-1)_{k_{1}}(b-1)_{k_{2}} u^{a-\left(k_{1}+1\right)} v^{b-\left(k_{2}+1\right)} w^{c-1}
$$

That is,

$$
\begin{aligned}
E(u, v, w)= & k_{1} \sum_{x=1}^{a-1}(x-1)_{k_{1}} u^{x-k_{1}} v^{\left[\frac{b x}{a}\right]} w^{\left[\frac{c x}{a}\right]} \\
& +(u-1) \sum_{x=1}^{a-1}(x-1)_{k_{1}+1} u^{x-\left(k_{1}+1\right)} v^{\left[\frac{b x}{a}\right]} w^{\left[\frac{c x}{a}\right]} \\
& +(v-1) \sum_{y=1}^{b-1}\left[\frac{a y}{b}\right]_{k_{1}} v^{y-1} w^{\left[\frac{c y}{b}\right]} u^{\left[\frac{a y}{b}\right]-k_{1}}+(w-1) \sum_{z=1}^{c-1}\left[\frac{a z}{c}\right]_{k_{1}} w^{z-1} u^{\left[\frac{a z}{c}\right]-k_{1}} v^{\left[\frac{b z}{c}\right]} \\
= & (a-1)_{k_{1}} u^{a-\left(k_{1}+1\right)} v^{b-1} w^{c-1} .
\end{aligned}
$$

Now we need to apply the same procedure to the function $E(u, v, w)$. If we calculate $k_{2}$ times the partial derivative of $E(u, v, w)$ with respect to $v$, we get

$$
G(u, v, w)=\frac{\partial^{k_{2}} E(u, v, w)}{\partial v^{k_{2}}}=(a-1)_{k_{1}}(b-1)_{k_{2}} u^{a-\left(k_{1}+1\right)} v^{b-\left(k_{2}+1\right)} w^{c-1}
$$


That is,

$$
\begin{aligned}
G(u, v, w)= & k_{1} \sum_{x=1}^{a-1}(x-1)_{k_{1}}\left[\frac{b x}{a}\right]_{k_{2}} u^{x-k_{1}} v^{\left[\frac{b x}{a}\right]-k_{2}} w^{\left[\frac{c x}{a}\right]} \\
& +(u-1) \sum_{x=1}^{a-1}(x-1)_{k_{1}+1}\left[\frac{b x}{a}\right]_{k_{2}} u^{x-\left(k_{1}+1\right)} v^{\left[\frac{b x}{a}\right]-k_{2}} w^{\left[\frac{c x}{a}\right]} \\
& +k_{2} \sum_{y=1}^{b-1}\left[\frac{a y}{b}\right]_{k_{1}}(y-1)_{k_{2}} v^{y-k_{2}} w^{\left[\frac{c y}{b}\right]} u^{\left[\frac{a y}{b}\right]-k_{1}} \\
& +(v-1) \sum_{y=1}^{b-1}\left[\frac{a y}{b}\right]_{k_{1}}(y-1)_{k_{2}+1} v^{y-\left(k_{2}+1\right)} w^{\left[\frac{c y}{b}\right]} u^{\left[\frac{a y}{b}\right]-k_{1}} \\
& +(w-1) \sum_{z=1}^{c-1}\left[\frac{a z}{c}\right]_{k_{1}}\left[\frac{b z}{c}\right]_{k_{2}} w^{z-1} u^{\left[\frac{a z}{c}\right]-k_{1}} v^{\left[\frac{b z}{c}\right]-k_{2}} \\
= & (a-1)_{k_{1}}(b-1)_{k_{2}} u^{a-\left(k_{1}+1\right)} v^{b-\left(k_{2}+1\right)} w^{c-1} .
\end{aligned}
$$

Finally, if we also take $k_{3}$ times partial derivative of $G(u, v, w)$, with respect to $w$, then we obtain the desired result.

Substituting $u_{1}=u_{2}=u_{3}=1$ into Definition 3, we get

$$
\begin{aligned}
\mathcal{F}\left(1,1,1 ; a, b, c ; k_{1}, k_{2}, k_{3}\right) \\
=k_{1} \sum_{x=1}^{a-1}(x-1)_{k_{1}-1}\left[\frac{b x}{a}\right]_{k_{2}}\left[\frac{c x}{a}\right]_{k_{3}}+k_{2} \sum_{y=1}^{b-1}(y-1)_{k_{2}-1}\left[\frac{a y}{b}\right]_{k_{1}}\left[\frac{c y}{b}\right]_{k_{3}} \\
\quad+k_{3} \sum_{z=1}^{c-1}(z-1)_{k_{3}-1}\left[\frac{a z}{c}\right]_{k_{1}}\left[\frac{b z}{c}\right]_{k_{2}}
\end{aligned}
$$

and by (22) we arrive at the following corollary.

\section{Corollary 3}

$$
\mathcal{F}\left(1,1,1 ; a, b, c ; k_{1}, k_{2}, k_{3}\right):=(a-1)_{k_{1}}(b-1)_{k_{2}}(c-1)_{k_{3}} .
$$

Corollary 4 If we substitute $k_{1}=1, k_{2}=0$, and $k_{3}=0$ into (23), we get

$$
\mathcal{F}(1,1,1 ; a, b, c ; 1,0,0)=a-1,
$$

if we substitute $k_{1}=0, k_{2}=1$, and $k_{3}=0$ into (23), we get

$$
\mathcal{F}(1,1,1 ; a, b, c ; 0,1,0)=b-1 \text {, }
$$

and finally if we substitute $k_{1}=0, k_{2}=0$, and $k_{3}=1$ into (23), we get

$$
\mathcal{F}(1,1,1 ; a, b, c ; 0,0,1)=c-1 \text {. }
$$


Remark 1 If we substitute $k_{1}=k_{2}=1, k_{3}=0$ in (23), then we have the following wellknown reciprocity law of the function $[x]$, which is very important to prove the Gauss law of quadratic reciprocity:

$$
\sum_{x=1}^{a-1}\left[\frac{b x}{a}\right]+\sum_{y=1}^{b-1}\left[\frac{a y}{b}\right]=(a-1)(b-1)
$$

(cf. $[6,7,14]$, and the references cited in each of these earlier works).

Remark 2 If we substitute $u=w=-1, v=1$ and $k_{1}=k_{3}=0, k_{2}=1$ into (21), we get

$$
\begin{aligned}
\mathcal{F}(-1,1,-1 ; a, b, c ; 0,1,0)= & -2 \sum_{x=1}^{a-1}\left[\frac{b x}{a}\right](-1)^{x+\left[\frac{c x}{a}\right]-1} \\
& +\sum_{y=1}^{b-1}(-1)^{\left[\frac{c y}{b}\right]+\left[\frac{a y}{b}\right]}-2 \sum_{z=1}^{c-1}\left[\frac{b z}{c}\right](-1)^{z+\left[\frac{a z}{c}\right]-1} .
\end{aligned}
$$

We also know from (22) that

$$
\mathcal{F}(-1,1,-1 ; a, b, c ; 0,1,0)=(b-1)(-1)^{a+c-2} .
$$

By combining (25) and (26), we get

$$
\begin{aligned}
& -2 \sum_{x=1}^{a-1}\left[\frac{b x}{a}\right](-1)^{x+\left[\frac{c x}{a}\right]-1}+\sum_{y=1}^{b-1}(-1)^{\left[\frac{c y}{b}\right]+\left[\frac{a y}{b}\right]}-2 \sum_{z=1}^{c-1}\left[\frac{b z}{c}\right](-1)^{z+\left[\frac{a z}{c}\right]-1} \\
& =(b-1)(-1)^{a+c-2}
\end{aligned}
$$

which gives us Theorem 2.1 in [14], so we have

$$
\mathcal{F}(-1,1,-1 ; a, b, c ; 0,1,0)=s_{4}\left(a c^{\prime}, b\right)-2 s_{5}\left(c b^{\prime}, a\right)-2 s_{3}\left(a b^{\prime}, c\right)
$$

or equivalently

$$
\mathcal{F}(-1,1,-1 ; a, b, c ; 0,1,0)=\frac{b-a c}{a c} .
$$

Remark 3 If we substitute $u=v=1, w=-1$ and $k_{1}=k_{2}=1, k_{3}=0$ into (21), we get

$$
\begin{aligned}
\mathcal{F}(1,1,-1 ; a, b, c ; 1,1,0)= & \sum_{x=1}^{a-1}(-1)^{\left[\frac{c x}{a}\right]}\left[\frac{b x}{a}\right]+\sum_{y=1}^{b-1}(-1)^{\left[\frac{c y}{b}\right]}\left[\frac{a y}{b}\right] \\
& -2 \sum_{z=1}^{c-1}(-1)^{z-1}\left[\frac{a z}{c}\right]\left[\frac{b z}{c}\right]
\end{aligned}
$$

we also know from (22) that

$$
\mathcal{F}(1,1,-1 ; a, b, c ; 1,1,0)=(a-1)(b-1)(-1)^{c-1}
$$


so if we use these two equations together, then we get

$$
\begin{aligned}
& \sum_{x=1}^{a-1}(-1)^{\left[\frac{c x}{a}\right]}\left[\frac{b x}{a}\right]+\sum_{y=1}^{b-1}(-1)^{\left[\frac{c y}{b}\right]}\left[\frac{a y}{b}\right]-2 \sum_{z=1}^{c-1}(-1)^{z-1}\left[\frac{a z}{c}\right]\left[\frac{b z}{c}\right] \\
& =(a-1)(b-1)(-1)^{c-1},
\end{aligned}
$$

which gives us Theorem 2.2 in [14], so we have

$$
\mathcal{F}(1,1,-1 ; a, b, c ; 1,1,0)=2 s_{2}\left(a b^{\prime}, c\right)-s_{1}\left(c b^{\prime}, a\right)-s_{3}\left(c a^{\prime}, b\right)
$$

or equivalently

$$
\mathcal{F}(1,1,-1 ; a, b, c ; 1,1,0)=-\frac{1}{2}+\frac{1}{2 c}\left(\frac{a}{c}+\frac{b}{c}\right) .
$$

Remark 4 If we substitute $u=-1, v=w=1$ and $k_{2}=1, k_{1}=k_{3}=0$ into (21), we get

$$
\mathcal{F}(-1,1,1 ; a, b, c ; 0,1,0)=-2 \sum_{x=1}^{a-1}(-1)^{x-1}\left[\frac{b x}{a}\right]+\sum_{y=1}^{b-1}(-1)^{\left[\frac{a y}{b}\right]}
$$

We also know from (22) that

$$
\mathcal{F}(-1,1,1 ; a, b, c ; 0,1,0)=(b-1)(-1)^{a-1}
$$

so if we use these two equations together, then we get

$$
-2 \sum_{x=1}^{a-1}(-1)^{x-1}\left[\frac{b x}{a}\right]+\sum_{y=1}^{b-1}(-1)^{\left[\frac{a y}{b}\right]}=(b-1)(-1)^{a-1}
$$

which gives us Theorem 2.3 in [14], so we have

$$
\mathcal{F}(-1,1,1 ; a, b, c ; 0,1,0)=2 s_{3}(b, a)-s_{4}(a, b)
$$

or equivalently

$$
\mathcal{F}(-1,1,1 ; a, b, c ; 0,1,0)=1-\frac{b}{a}
$$

Remark 5 If we substitute $u=v=-1, w=1$ and $k_{1}=k_{2}=0, k_{3}=1$ into (21), we get

$$
\begin{aligned}
\mathcal{F}( & -1,-1,1 ; a, b, c ; 0,0,1) \\
& =-2 \sum_{x=1}^{a-1}(-1)^{x+\left[\frac{b x}{a}\right]-1}\left[\frac{c x}{a}\right]-2 \sum_{y=1}^{b-1}(-1)^{y+\left[\frac{a y}{b}\right]-1}\left[\frac{c y}{b}\right]+\sum_{z=1}^{c-1}(-1)^{\left[\frac{a z}{c}\right]+\left[\frac{b z}{c}\right]}
\end{aligned}
$$

we also know from (22) that

$$
\mathcal{F}(-1,-1,1 ; a, b, c ; 0,0,1)=(c-1)(-1)^{a+b-2}
$$


so if we use these two equations together, then we get

$$
-2 \sum_{x=1}^{a-1}(-1)^{x+\left[\frac{b x}{a}\right]-1}\left[\frac{c x}{a}\right]-2 \sum_{y=1}^{b-1}(-1)^{y+\left[\frac{a y}{b}\right]-1}\left[\frac{c y}{b}\right]+\sum_{z=1}^{c-1}(-1)^{\left[\frac{a z}{c}\right]+\left[\frac{b z}{c}\right]}=(c-1)(-1)^{a+b-2},
$$

which gives us Theorem 2.4 in [14], so we have

$$
\mathcal{F}(-1,-1,1 ; a, b, c ; 0,0,1)=s_{4}\left(a b^{\prime}, c\right)+2 s_{5}\left(b c^{\prime}, a\right)-s_{1}\left(a c^{\prime}, b\right)
$$

or equivalently

$$
\mathcal{F}(-1,-1,1 ; a, b, c ; 0,0,1)=-1+\frac{c}{a b} \text {. }
$$

Remark 6 If we substitute $w=v=1, u=-1$ and $k_{1}=0, k_{2}=k_{3}=1$ into (21), we get

$$
\begin{aligned}
\mathcal{F}(-1,1,1 ; a, b, c ; 0,1,1)= & -2 \sum_{x=1}^{a-1}(-1)^{x-1}\left[\frac{b x}{a}\right]\left[\frac{c x}{a}\right] \\
& +\sum_{y=1}^{b-1}(-1)^{\left[\frac{a y}{b}\right]}\left[\frac{c y}{b}\right]+\sum_{z=1}^{c-1}(-1)^{\left[\frac{a z}{c}\right]}\left[\frac{b z}{c}\right]
\end{aligned}
$$

we also know from (22) that

$$
\mathcal{F}(-1,1,1 ; a, b, c ; 0,1,1)=(b-1)(c-1)(-1)^{a-1},
$$

so if we use these two equations together, then we get

$$
-2 \sum_{x=1}^{a-1}(-1)^{x-1}\left[\frac{b x}{a}\right]\left[\frac{c x}{a}\right]+\sum_{y=1}^{b-1}(-1)^{\left[\frac{a y}{b}\right]}\left[\frac{c y}{b}\right]+\sum_{z=1}^{c-1}(-1)^{\left[\frac{a z}{c}\right]}\left[\frac{b z}{c}\right]=(b-1)(c-1)(-1)^{a-1}
$$

which gives us Theorem 2.5 in [14], so we have

$$
\mathcal{F}(-1,1,1 ; a, b, c ; 0,1,1)=2 s_{2}\left(c b^{\prime}, a\right)-s_{1}\left(c a^{\prime}, b\right)-s_{3}\left(c a^{\prime}, b\right)
$$

or equivalently

$$
\mathcal{F}(-1,1,1 ; a, b, c ; 0,1,1)=-\frac{1}{2}+\frac{1}{2 a}\left(\frac{c}{b}+\frac{b}{c}\right) .
$$

We can also have some results from [11] by using the same method as follows.

Remark 7 If we substitute $u=1, v=w=-1$ and $k_{1}=1, k_{2}=k_{3}=0$ into (21), we get

$$
\begin{aligned}
\mathcal{F} & (1,-1,-1 ; a, b, c ; 1,0,0) \\
& =\sum_{x=1}^{a-1}(-1)^{\left[\frac{b x}{a}\right]+\left[\frac{c x}{a}\right]}-2 \sum_{y=1}^{b-1}(-1)^{y+\left[\frac{c y}{b}\right]}\left[\frac{a y}{b}\right]-2 \sum_{z=1}^{c-1}(-1)^{z+\left[\frac{b z}{c}\right]-1}\left[\frac{a z}{c}\right]
\end{aligned}
$$


we also know from (22) that

$$
\mathcal{F}(1,-1,-1 ; a, b, c ; 1,0,0)=(a-1)(-1)^{b+c},
$$

so if we use these two equations together, then we get

$$
\begin{aligned}
& \sum_{x=1}^{a-1}(-1)^{\left[\frac{b x}{a}\right]+\left[\frac{c x}{a}\right]}-2 \sum_{y=1}^{b-1}(-1)^{y+\left[\frac{c y}{b}\right]}\left[\frac{a y}{b}\right]-2 \sum_{z=1}^{c-1}(-1)^{z+\left[\frac{b z}{c}\right]-1}\left[\frac{a z}{c}\right] \\
& \quad=(a-1)(-1)^{b+c},
\end{aligned}
$$

which gives us Theorem 3.3 in [11], so we have

$$
\mathcal{F}(1,-1,-1 ; a, b, c ; 1,0,0)=s_{3}\left(a b^{\prime}, c\right)+s_{1}\left(c a^{\prime}, b\right)-\frac{1}{2} s_{4}\left(b c^{\prime}, a\right)
$$

or equivalently

$$
\mathcal{F}(1,-1,-1 ; a, b, c ; 1,0,0)=\frac{1}{2}-\frac{a}{2 b c},
$$

where $a$ and $a^{\prime}$ are even and $c c^{\prime} \equiv 1 \quad(\bmod 2 a)$.

Remark 8 If we substitute $u=w=1, v=-1$ and $k_{1}=k_{3}=1, k_{2}=0$ into (21), we get

$$
\begin{aligned}
\mathcal{F} & (1,-1,1 ; a, b, c ; 1,0,1) \\
& =\sum_{x=1}^{a-1}(-1)^{\left[\frac{b x}{a}\right]}\left[\frac{c x}{a}\right]+2 \sum_{y=1}^{b-1}(-1)^{y}\left[\frac{a y}{b}\right]\left[\frac{c y}{b}\right]+\sum_{z=1}^{c-1}(-1)^{\left[\frac{b z}{c}\right]}\left[\frac{a z}{c}\right] .
\end{aligned}
$$

From (22), we see that

$$
\mathcal{F}(1,-1,1 ; a, b, c ; 1,0,1)=(a-1)(c-1)(-1)^{b-1} .
$$

Therefore

$$
\begin{aligned}
& \sum_{x=1}^{a-1}(-1)^{\left[\frac{b x}{a}\right]}\left[\frac{c x}{a}\right]+2 \sum_{y=1}^{b-1}(-1)^{y}\left[\frac{a y}{b}\right]\left[\frac{c y}{b}\right]+\sum_{z=1}^{c-1}(-1)^{\left[\frac{b z}{c}\right]}\left[\frac{a z}{c}\right] \\
& =(a-1)(c-1)(-1)^{b-1},
\end{aligned}
$$

which gives us Theorem 3.4 in [11], so we have

$$
\mathcal{F}(1,-1,1 ; a, b, c ; 1,0,1)=s_{1}\left(b c^{\prime}, a\right)-2 s_{2}\left(c a^{\prime}, b\right)+s_{3}\left(a b^{\prime}, c\right)
$$

or equivalently

$$
\mathcal{F}(1,-1,1 ; a, b, c ; 1,0,1)=\frac{1}{2}-\frac{1}{2}\left(\frac{a}{b c}+\frac{c}{a b}\right),
$$

where $b$ is even. 
$\operatorname{Remark} 9$ If we substitute $u=v=w=1$, and $k_{1}=k_{2}=k_{3}=1$, into (21), we get

$$
\begin{aligned}
\mathcal{F}(1,1,1 ; a, b, c ; 1,1,1) \\
=\sum_{x=1}^{a-1}\left[\frac{b x}{a}\right]\left[\frac{c x}{a}\right]+\sum_{y=1}^{b-1}\left[\frac{a y}{b}\right]\left[\frac{c y}{b}\right]+\sum_{z=1}^{c-1}\left[\frac{a z}{c}\right]\left[\frac{b z}{c}\right]
\end{aligned}
$$

we also know from (22) that

$$
\mathcal{F}(1,1,1 ; a, b, c ; 1,1,1)=(a-1)(b-1)(c-1) .
$$

Therefore

$$
\sum_{x=1}^{a-1}\left[\frac{b x}{a}\right]\left[\frac{c x}{a}\right]+\sum_{y=1}^{b-1}\left[\frac{a y}{b}\right]\left[\frac{c y}{b}\right]+\sum_{z=1}^{c-1}\left[\frac{a z}{c}\right]\left[\frac{b z}{c}\right]=(a-1)(b-1)(c-1)
$$

(cf. $[11,(4.1)]$, and the references cited in each of these earlier works).

By the mathematical induction method, we shall generalize Theorem 6 . But first we need a new definition.

Definition 4 Let $u_{1}, \ldots, u_{n}$ be indeterminants. Let $a_{1}, \ldots, a_{n}$ be positive integers, relatively prime in pairs, and let $k_{1}, \ldots, k_{n}$ be positive natural numbers, which are the orders of the derivatives of $u_{1}, \ldots, u_{n}$, respectively. Then the polynomial $\mathcal{F}\left(u_{1}, \ldots, u_{n} ; a_{1}, \ldots, a_{n}\right.$; $\left.k_{1}, \ldots, k_{n}\right)$ is defined by

$$
\begin{aligned}
& \mathcal{F}\left(u_{1}, \ldots, u_{n} ; a_{1}, \ldots, a_{n} ; k_{1}, \ldots, k_{n}\right) \\
& =\sum_{l=1}^{n}\left(u_{l}-1\right) \sum_{x_{l}=1}^{a_{l}-1}\left(x_{l}-1\right)_{k_{l}} \prod_{m=1, m \neq l}^{n}\left[\frac{a_{m} x_{l}}{a_{l}}\right]_{k_{m}} u_{m}^{\left[\frac{a_{m} x_{l}}{a_{l}}\right]} u_{l}^{x_{l}-1} \\
& +\sum_{l=1}^{n} k_{l} \sum_{x_{l}=1}^{a_{l}-1}\left(x_{l}-1\right)_{k_{l}-1} \prod_{m=1, m \neq l}^{n}\left[\frac{a_{m} x_{l}}{a_{l}}\right]_{k_{m}} u_{m}^{\left[\frac{a_{m} x_{l}}{a_{l}}\right]} u_{l}^{x_{l}} .
\end{aligned}
$$

Now we can give the generalization of Theorem 6 as follows.

Theorem 7 Let $u_{1}, u_{2}, \ldots, u_{n}$ be indeterminants. Let $a_{1}, a_{2}, \ldots, a_{n}$ be positive integers, relatively prime in pairs, and let $k_{1}, k_{2}, \ldots, k_{n}$ be positive integers. For $n \geq 2$, the following identity holds true:

$$
\mathcal{F}\left(u_{1}, \ldots, u_{n} ; a_{1}, \ldots, a_{n} ; k_{1}, \ldots, k_{n}\right)=\prod_{m=1}^{n} u_{m}^{a_{m}-1}\left(a_{m}-1\right)_{k_{m}} .
$$

Remark 10 If we substitute $n=3$ in Theorem 7, then Theorem 7 reduces to Theorem 6 .

Corollary 5 Substituting $u_{1}=u_{2}=\cdots=u_{n}=1$ and $k_{1}=k_{2}=\cdots=k_{n}=1$ into Definition 4 and Theorem 7, we arrive at 


$$
\begin{aligned}
& \sum_{x_{1}=1}^{a_{1}-1}\left[\frac{a_{2} x_{1}}{a_{1}}\right]\left[\frac{a_{3} x_{1}}{a_{1}}\right] \cdots\left[\frac{a_{n} x_{1}}{a_{1}}\right]+\sum_{x_{2}=1}^{a_{2}-1}\left[\frac{a_{1} x_{2}}{a_{2}}\right]\left[\frac{a_{3} x_{2}}{a_{2}}\right] \cdots\left[\frac{a_{n} x_{2}}{a_{2}}\right] \\
& +\cdots+\sum_{x_{n}=1}^{a_{n}-1}\left[\frac{a_{1} x_{n}}{a_{n}}\right]\left[\frac{a_{2} x_{n}}{a_{n}}\right] \cdots\left[\frac{a_{n-1} x_{n}}{a_{n}}\right] \\
& =\left(a_{1}-1\right)\left(a_{2}-1\right) \cdots\left(a_{n}-1\right) .
\end{aligned}
$$

A proof of Corollary 5 was given by Beck [6, Corollary 3.1].

Remark 11 Substituting $n=3$ into (27), we easily have

$$
\begin{aligned}
& \sum_{x_{1}=1}^{a_{1}-1}\left[\frac{a_{2} x_{1}}{a_{1}}\right]\left[\frac{a_{3} x_{1}}{a_{1}}\right]+\sum_{x_{2}=1}^{a_{2}-1}\left[\frac{a_{1} x_{2}}{a_{2}}\right]\left[\frac{a_{3} x_{2}}{a_{2}}\right]+\sum_{x_{3}=1}^{a_{3}-1}\left[\frac{a_{1} x_{3}}{a_{3}}\right]\left[\frac{a_{2} x_{3}}{a_{3}}\right] \\
& =\left(a_{1}-1\right)\left(a_{2}-1\right)\left(a_{3}-1\right) .
\end{aligned}
$$

By substituting $[x]=x-((x))-\frac{1}{2}$ into the above equation, one can arrive at the reciprocity law of the Dedekind-Rademacher sums:

$$
\begin{aligned}
\sum_{x_{1}=1}^{a_{1}-1} & \left(\left(\frac{a_{2} x_{1}}{a_{1}}\right)\right)\left(\left(\frac{a_{3} x_{1}}{a_{1}}\right)\right)+\sum_{x_{2}=1}^{a_{2}-1}\left(\left(\frac{a_{1} x_{2}}{a_{2}}\right)\right)\left(\left(\frac{a_{3} x_{2}}{a_{2}}\right)\right) \\
& +\sum_{x_{3}=1}^{a_{3}-1}\left(\left(\frac{a_{1} x_{3}}{a_{3}}\right)\right)\left(\left(\frac{a_{2} x_{3}}{a_{3}}\right)\right) \\
= & -\frac{1}{4}+\frac{1}{12}\left(\frac{a_{1}}{a_{2} a_{3}}+\frac{a_{2}}{a_{1} a_{3}}+\frac{a_{2}}{a_{1} a_{2}}\right)
\end{aligned}
$$

$(c f .[6,12]$, and the references cited in each of these earlier works).

Substituting $u_{1}=u_{2}=\cdots=u_{n}=1$ into Definition 4 and Theorem 7, then we arrive at the following result.

Corollary 6 Let $a_{1}, a_{2}, \ldots, a_{n}$ be positive integers, relatively prime in pairs, and let $k_{1}, k_{2}, \ldots, k_{n}$ be positive integers. Then we have

$$
\sum_{l=1}^{n} k_{l} \sum_{x_{l}=1}^{a_{l}-1}\left(x_{l}-1\right)_{k_{l}-1} \prod_{m=1, m \neq l}^{n}\left[\frac{a_{m} x_{l}}{a_{l}}\right]_{k_{m}}=\prod_{m=1}^{n}\left(a_{m}-1\right)_{k_{m}} .
$$

Remark 12 Setting $k_{1}=k_{2}=\cdots=k_{n}=1$ in (28), we obtain the following well-known identity, which was proved by Berndt and Dieter [7, p.212, Corollary 3.5]:

$$
\sum_{l=1}^{n} \sum_{x_{l}=1}^{a_{l}-1} \prod_{m=1, m \neq l}^{n}\left[\frac{a_{m} x_{l}}{a_{l}}\right]=\prod_{m=1}^{n}\left(a_{m}-1\right)
$$

where $a_{1}, a_{2}, \ldots, a_{n}$ be positive integers, relatively prime in pairs ( $c f$. also [6, Corollary 3.1]). Note that this result was also obtained in Corollary 5. 
Substituting $u_{1}=u_{2}=\cdots=u_{n}=-1$ into Definition 4 and Theorem 7, we obtain the following corollary.

\section{Corollary 7}

$$
\begin{aligned}
& \sum_{l=1}^{n} \sum_{x_{l}=1}^{a_{l}-1}\left(2\left(x_{l}-1\right)_{k_{l}}+k_{l}\left(x_{l}-1\right)_{k_{l}-1}\right) \prod_{m=1, m \neq l}^{n}\left[\frac{a_{m} x_{l}}{a_{l}}\right]_{k_{m}}(-1)^{x_{l}+\left[\frac{a_{m} x_{l}}{a_{l}}\right]} \\
& \quad=\prod_{m=1}^{n}(-1)^{a_{m}-1}\left(a_{m}-1\right)_{k_{m}} .
\end{aligned}
$$

Remark 13 Substituting $n=2$ and $k_{1}=k_{2}=1$, in Corollary 7, then we arrive at

$$
\begin{aligned}
& \sum_{x_{1}=1}^{a_{1}-1}\left(2 x_{1}-1\right)(-1)^{x_{1}+\left[\frac{a_{2} x_{1}}{a_{1}}\right]}\left[\frac{a_{2} x_{1}}{a_{1}}\right]+\sum_{x_{2}=1}^{a_{2}-1}\left(2 x_{2}-1\right)(-1)^{x_{2}+\left[\frac{a_{1} x_{2}}{a_{2}}\right]}\left[\frac{a_{1} x_{2}}{a_{2}}\right] \\
& \quad=(-1)^{a_{1}+a_{2}}\left(a_{1}-1\right)\left(a_{2}-1\right) .
\end{aligned}
$$

We are now ready to give a proof of Theorem 4.

Proof of Theorem 4 Substituting $k_{1}=k_{2}=\cdots=k_{n}=1$ into (29), we obtain

$$
\sum_{l=1}^{n} \sum_{x_{l}=1}^{a_{l}-1}\left(2 x_{l}-1\right) \prod_{m=1, m \neq l}^{n}(-1)^{x_{l}+\left[\frac{a_{m} x_{l}}{a_{l}}\right]}\left[\frac{a_{m} x_{l}}{a_{l}}\right]=\prod_{m=1}^{n}(-1)^{a_{m}-1}\left(a_{m}-1\right) .
$$

Combining the above equation with (17), we get

$$
\begin{aligned}
& Y_{n-1}\left(a_{2}, a_{3}, \ldots, a_{n} ; a_{1}\right)+Y_{n-1}\left(a_{1}, a_{3}, \ldots, a_{n} ; a_{2}\right)+\cdots+Y_{n-1}\left(a_{1}, a_{2}, \ldots, a_{n-1} ; a_{n}\right) \\
& \quad=\prod_{m=1}^{n}(-1)^{a_{m}-1}\left(a_{m}-1\right) .
\end{aligned}
$$

Hence, we arrive at the desired result.

\section{Competing interests}

The authors declare that they have no competing interests.

Authors' contributions

All authors contributed equally to the writing of this paper. All authors read and approved the final manuscript.

\section{Author details}

'Department of Mathematics, Uludag University, Gorukle, Bursa 16059, Turkey. ${ }^{2}$ Department of Mathematics, Akdeniz University, Antalya, 07058, Turkey.

\section{Acknowledgements}

The authors are supported by the research funds of Akdeniz University and Uludag University (Uludag University project numbers are 201424 and 201220). 


\section{References}

1. Berndt, BC: Analytic Eisenstein series, Theta-functions, and series relations in the spirit of Ramanujan. J. Reine Angew. Math. 303/304, 332-365 (1978)

2. Goldberg, LA: Transformation of Theta-functions and analogues of Dedekind sums. Thesis, University of Illinois Urbana (1981)

3. Simsek, Y: Relations between theta-functions Hardy sums Eisenstein series and Lambert series in the transformation formula of $\log \eta_{g, h}(z)$. J. Number Theory 99, 338-360 (2003)

4. Apostol, TM: Modular Functions and Dirichlet Series in Number Theory. Springer, Berlin (1976)

5. Apostol, TM, Vu, TH: Elementary proofs of Berndt's reciprocity laws. Pac. J. Math. 98, 17-23 (1982)

6. Beck, M: Geometric proofs of polynomial reciprocity laws of Carlitz, Berndt, and Dieter. In: Diophantine Analysis and Related Fields. Sem. Math. Sci., vol. 35, pp. 11-18 (2006)

7. Berndt, BC, Dieter, U: Sums involving the greatest integer function and Riemann Stieltjes integration. J. Reine Angew. Math. 337, 208-220 (1982)

8. Berndt, BC, Goldberg, LA: Analytic properties of arithmetic sums arising in the theory of the classical Theta-functions. SIAM J. Math. Anal. 15, 143-150 (1984)

9. Carlitz, L: Some polynomials associated with Dedekind sums. Acta Math. Acad. Sci. Hung. 26, 311-319 (1975)

10. Hardy, GH: On certain series of discontinues functions connected with the modular functions. Q. J. Math. 36, 93-123 (1905)

11. Pettet, MR, Sitaramachandrarao, R: Three-term relations for Hardy sums. J. Number Theory 25, 328-339 (1989)

12. Rademacher, H: Generalization of the reciprocity formula for Dedekind sums. Duke Math. J. 21, $391-397$ (1954)

13. Simsek, Y: On generalized Hardy sums $s_{5}(h, k)$. Ukr. Math. J. 56(10), 1434-1440 (2004)

14. Simsek, Y: Theorems on three-term relations for Hardy sums. Turk. J. Math. 22, 153-162 (1998)

15. Simsek, Y: A note on Dedekind sums. Bull. Calcutta Math. Soc. 85, 567-572 (1993)

16. Simsek, Y: On analytic properties and character analogs of Hardy sums. Taiwan. J. Math. 13, 253-268 (2009)

17. Sitaramachandrarao, R: Dedekind and Hardy sums. Acta Arith. 48, 325-340 (1987)

10.1186/1687-1847-2014-283

Cite this article as: Cetin et al.: Some special finite sums related to the three-term polynomial relations and their applications. Advances in Difference Equations 2014, 2014:283

\section{Submit your manuscript to a SpringerOpen ${ }^{\ominus}$ journal and benefit from:}

- Convenient online submission

- Rigorous peer review

- Immediate publication on acceptance

- Open access: articles freely available online

- High visibility within the field

- Retaining the copyright to your article 